Michą Olech (Wrocław)

\title{
NONUNIQUENESS OF STEADY STATES IN ANNULAR DOMAINS FOR STREATER EQUATIONS
}

Abstract. Models introduced by R. F. Streater describe the evolution of the density and temperature of a cloud of self-gravitating particles. We study nonuniqueness of steady states in annular domains in $\mathbb{R}^{d}, d \geq 2$.

1. History of the problem and physical motivation. The system we will deal with is of the form

$$
\begin{aligned}
u_{t} & =-\nabla \cdot \mathbf{j}, \\
(u \theta)_{t} & =\nabla \cdot(\lambda \nabla \theta)-\nabla \cdot(\theta \mathbf{j})-\nabla\left(\phi+\phi_{0}\right) \cdot \mathbf{j}, \\
\Delta \phi & =u,
\end{aligned}
$$

where $\mathbf{j}=-\kappa\left(\nabla u+\frac{u}{\theta}\left(\nabla \phi+\nabla \phi_{0}\right)\right)$. We will supplement this system with the following boundary conditions for $u$ and $\theta$ :

$$
\begin{aligned}
\frac{\partial u}{\partial \nu}+\frac{u}{\theta}\left(\partial_{\nu} \phi+\partial_{\nu} \phi_{0}\right) & =0, \\
\frac{\partial \theta}{\partial \nu} & =0,
\end{aligned}
$$

and we will consider two kinds of boundary conditions for $\phi$ :

$$
\begin{gathered}
\text { either the Dirichlet condition: } \phi=0 \text { on } \partial \Omega, \\
\text { or the "free" condition: } \phi=E_{d} * u_{\Omega},
\end{gathered}
$$

where $u_{\Omega}(x)=u(x)$ in $\Omega$ and vanishes outside $\Omega$, and $(f * g)(x)$ means the convolution $\int_{\Omega} f(x-y) g(y) d y$ ( $E_{d}$ denotes the fundamental solution of the Laplacian in $\mathbb{R}^{d}$ ). We call (1) with (2)-(4) the Dirichlet problem, and (2), (3), (5) the "free" problem.

These systems describe the evolution of the density $u(x, t) \geq 0$ and the temperature $\theta(x, t)>0$ in a cloud of self-gravitating particles under the

2000 Mathematics Subject Classification: 35Q, 35J60, 82C21.

Key words and phrases: Streater's models, existence and uniqueness of stationary solutions, Poisson-Boltzmann-Emden equation, phase plane method. 
external potential $\phi_{0}(x)$ with self-induced potential $\phi$. The coefficients $\kappa$ and $\lambda$ are nonnegative functions of $x, u, \theta$ and $\phi$. Furthermore, they can vanish only when $\theta=0$.

In [9] this system has been derived by Streater from the Smoluchowski equation (see [8]). This was generalized in [2] to the case of gravitational and electric self-interactions.

Some results on existence and uniqueness of solutions for (1) can be found e.g. in [3] and in the references therein.

2. Steady states equation. Following [1] we begin by recalling basic properties of solutions to (1)-(5).

Proposition 2.1. For sufficiently smooth solutions of the Dirichlet and the "free" problem we have:

(i) the mass $M=\int_{\Omega} u d x$ and the energy $E=\int_{\Omega} u\left(\theta+\phi_{0}+\frac{1}{2} \phi\right) d x$ of the system are constant in time,

(ii) the entropy

$$
W=\int_{\Omega} u \log \left(\frac{u}{\theta}\right) d x
$$

does not increase in time.

Proof. The first assertion follows easily from the boundary conditions, the Gauss-Green theorem and (1). For the entropy, a direct calculation shows that

$$
\begin{aligned}
\frac{d W}{d t} & =\frac{d}{d t} \int_{\Omega} u \log \frac{u}{\theta} d x=\int_{\Omega} u_{t} \log \frac{u}{\theta} d x+\int_{\Omega}\left(\frac{u}{\theta}\right)_{t} \cdot \frac{\theta}{u} d x \\
& =-\int_{\Omega} \frac{1}{\theta} \nabla \cdot(\lambda \nabla \theta) d x-\int_{\Omega}\left(\frac{\nabla u}{u}+\frac{1}{\theta}\left(\nabla \phi+\nabla \phi_{0}\right) \cdot \mathbf{j}\right) d x \\
& =-\int_{\Omega} \lambda \frac{|\nabla \theta|^{2}}{\theta^{2}} d x-\int_{\Omega} \kappa u\left|\frac{\nabla u}{u}+\frac{1}{\theta}\left(\nabla \phi+\nabla \phi_{0}\right)\right|^{2} d x \leq 0,
\end{aligned}
$$

which completes the proof.

For a steady state $\left(u_{\infty}, \theta_{\infty}\right)$ (i.e. a solution independent of time), the entropy of the system is constant. From this trivial fact and from (6) we deduce that

$$
\nabla u_{\infty}+\frac{u_{\infty}}{\theta_{\infty}} \nabla\left(\phi_{\infty}+\phi_{0}\right)
$$

vanishes a.e. in $\Omega$, and the temperature is constant: $\theta_{\infty}=$ const. Multiplying by the nonzero factor $e^{\left(\phi_{\infty}+\phi_{0}\right) / \theta_{\infty}}$ and integrating, we obtain the equation 
for steady states

$$
u_{\infty}=\frac{M e^{-\left(\phi_{\infty}+\phi_{0}\right) / \theta_{\infty}}}{\int_{\Omega} e^{-\left(\phi_{\infty}+\phi_{0}\right) / \theta_{\infty}} d x} .
$$

For simplicity of notation we write $\phi_{\infty}:=\phi, \theta_{\infty}:=\theta, u_{\infty}:=u$, and we will consider only the case $\phi_{0} \equiv 0$.

As shown in [1], if we put $\phi=\theta \psi$ in (7), the energy relation becomes

$$
E=\int_{\Omega} u\left(\theta+\frac{1}{2} \phi\right) d x=\theta \int_{\Omega} u d x+\frac{\theta^{2}}{2} \int_{\Omega} u \psi d x=\theta M+\frac{\theta^{2}}{2} \int_{\Omega} \psi \Delta \psi d x .
$$

Then we can replace the problem of determination of steady states with prescribed mass $M>0$ and energy $E$ by the problem

$$
\left(\frac{E}{M^{2}}\right) m^{2}=m-\frac{1}{2} \int_{\Omega} \psi \Delta \psi d x=\mathcal{E}(m, \psi)
$$

where $m=M / \theta$ and $\psi$ solves the Poisson-Boltzmann-Emden equation

$$
\begin{array}{ll}
\Delta \psi=m \frac{e^{-\psi}}{\int_{\Omega} e^{-\psi} d x} & \text { in } \Omega, \\
\psi=0 & \text { on } \partial \Omega
\end{array}
$$

for the Dirichlet problem, and

for the "free" problem.

$$
\psi=\frac{m}{\int_{\Omega} e^{-\psi} d x} E_{d} * e^{-\psi}
$$

3. $k$-symmetric solutions for the Dirichlet problem. In this section we will deal with the two-dimensional Dirichlet problem (8) in an annular domain. Recall that the existence of radial solutions for all values of $m>0$ was proved in [4]. Here we show the existence of nonradial solutions. Moreover, we show nonuniqueness of solutions and we study their exact shape.

To do this we take advantage of a reasoning from [7], concerning the existence, for $\lambda \geq 0$, of solutions of the problem

$$
\begin{aligned}
\Delta v+\lambda e^{v}=0 & \text { in } \Omega, \\
v=0 & \text { on } \partial \Omega .
\end{aligned}
$$

Without loss of generality, we set $\Omega=\left\{x \in \mathbb{R}^{2}: 0<a<|x|<1\right\}$. Let $T_{k} x$ be the rotation of point $x$ about the origin through $2 \pi / k$, where $k=1,2,3, \ldots$, and let

$$
\begin{aligned}
V_{k} & =\left\{v \in H_{0}^{1}(\Omega): v\left(T_{k} x\right)=v(x) \text { a.e. in } \Omega\right\}, \\
V_{\infty} & =\left\{v \in H_{0}^{1}(\Omega): v \text { is radial }\right\} .
\end{aligned}
$$

Then $V_{k}$ and $V_{\infty}$ are closed subspaces of $H_{0}^{1}(\Omega)$. 
It is evident that for $v \in V_{k}(k=\infty, 1,2,3, \ldots)$ and $\lambda \in \mathbb{R}$, if $w$ solves

$$
\begin{aligned}
& \Delta w+\lambda e^{v}=0 \quad \text { in } \Omega, \\
& w=0 \quad \text { on } \partial \Omega \text {, }
\end{aligned}
$$

then $w$ also belongs to $V_{k}$.

Next we introduce two functionals

$$
\begin{aligned}
& \Phi(v)=\int_{\Omega} e^{v(x)} d x, \\
& J(v)=\frac{1}{2} \int_{\Omega}|\nabla v(x)|^{2} d x,
\end{aligned}
$$

and define for every $k \in \mathbb{N}$ a subset of $V_{k}$ by

$$
K_{k, \mu}=\left\{v \in V_{k}: \Phi(v)=\mu\right\} \quad \text { for } \mu \in \mathbb{R} .
$$

Let $j_{k}[\mu]=\inf _{v \in K_{k, \mu}} J(v)$ whenever $K_{k, \mu} \neq \emptyset$. We will say that $v$ is $k$ symmetric when $v \in V_{k}$ and $v \notin V_{l}$ for any $l>k$.

Our goal is to prove

TheOREM 3.1. For every $k \in \mathbb{N}$ there exists a $k$-symmetric solution of the Dirichlet problem (8) for some $m>0$.

In [7] the following is proved:

THEOREM 3.2. For every $k=\infty, 1,2,3, \ldots$ such that $K_{k, \mu} \subset V_{k}$ is not empty there exists $v_{\mu} \in K_{k, \mu}$ which minimizes (12) (i.e. $j_{k}[\mu]=J\left(v_{\mu}\right)$ ). Moreover, the minimizer $v_{\mu}$ solves

$$
\begin{aligned}
\Delta v_{\mu}+\lambda_{\mu} e^{v_{\mu}}=0 & \text { in } \Omega, \\
v_{\mu}=0 & \text { on } \partial \Omega,
\end{aligned}
$$

for some parameter $\lambda_{\mu} \in \mathbb{R}$. $3.4])$.

We also need two technical lemmas (for details see [7, Lemmas 3.3 and

Lemma 3.3. For every $k \in \mathbb{N}$ and sufficiently large $\mu$ we have

$$
j_{k}[\mu]<j_{\infty}[\mu] .
$$

Lemma 3.4. For every $k \in \mathbb{N}$ we have

$$
j_{1}[\mu] \leq j_{2}[\mu] \leq \cdots \leq j_{k}[\mu] \leq \cdots \leq j_{\infty}[\mu] .
$$

Moreover, $j_{k}[\mu]<j_{\infty}[\mu]$ implies

$$
j_{1}[\mu]<j_{2}[\mu]<\cdots<j_{k}[\mu] .
$$

Notice that for $\mu>|\Omega|$ we have $\lambda>0$ and the solution $v \in K_{k, \mu}$ of the minimization problem also solves (10). Indeed, suppose, on the contrary, 
that $\lambda<0$. From the maximum principle for elliptic problems we have $v \leq 0$ in $\Omega$. Then

$$
\mu=\int_{\Omega} e^{v} d x \leq \int_{\Omega} 1 d x=|\Omega|,
$$

which contradicts the assumption $\mu>|\Omega|$.

Now we recall the main theorem from [7, Th. 3.5] with its proof.

THEOREM 3.5. For all $k \in \mathbb{N}$ there exists $\mu_{k}$ such that for all $\mu>\mu_{k}$ the problem (10) has a k-symmetric solution. This solution satisfies

$$
\int_{\Omega} e^{v} d x=\mu .
$$

Proof. By Lemma 3.3 and Theorem 3.2 there exists $v=v_{k, \mu} \in V_{k}$ such that $J(v)=j_{k}[\mu]<j_{\infty}[\mu]$ for some $\mu$ sufficiently large. It suffices to show that $v$ is $k$-symmetric.

Suppose, contrary to our claim, that there exists a natural number $l>k$ such that $v \in V_{l}$. Then

$$
j_{l}[\mu] \leq J(v)=j_{k}[\mu]<j_{\infty}[\mu],
$$

which contradicts Lemma 3.4.

Now let us put $v \mapsto-v$ in (10). Then we obtain

$$
\begin{array}{clrl}
\Delta v & =\lambda e^{-v} & & \text { in } \Omega, \\
v & =0 & & \text { on } \partial \Omega,
\end{array}
$$

$\Phi(-v)=\Phi_{1}(v)=\int_{\Omega} e^{-v} d x$, and (8) takes the form

$$
\begin{aligned}
\Delta v & =\frac{m}{\Phi_{1}(v)} e^{-v} & & \text { in } \Omega, \\
v & =0 & & \text { on } \partial \Omega .
\end{aligned}
$$

Proof of Theorem 3.1. From Theorem 3.5 we have a $k$-symmetric solution to the problem (10) for sufficiently large values of $\mu=\Phi_{1}(v)$. This solution solves (8) with $m=\lambda \mu$.

Since (8) has radial solutions for all $m>0$ (see [4]), the following corollary is obvious:

COROllaRY 3.6. There is nonuniqueness of solutions for the problem (8) in a two-dimensional annulus for some values of $m>0$.

4. Radial solutions for the "free" problem. Now we will analyze (9) using dynamical systems methods. An example of use of this method is in [3].

Existence results for solutions to the problem (9) can be found in [3] and the references therein. 
Our main goal in this section is to prove

THEOREM 4.1. For any a and some values of $A$ and $m$, the "free" problem (9) has at least two radial solutions in $\Omega_{a}^{A}=\left\{x \in \mathbb{R}^{d}: 0<a<|x|<A\right.$ $<\infty\}$ for $3 \leq d \leq 9$.

Assume that $\psi$ is radial. Abusing the notation, we set $\psi(x)=\psi(|x|)=$ $\psi(r)$. Then $\psi$ solves

$$
\psi=\frac{m}{\int_{\Omega} e^{-\psi} d x} E_{d} * e^{-\psi}
$$

in the annulus $\Omega_{a}^{A}$.

It is obvious that $\psi$ also solves

$$
\Delta \psi=m \frac{e^{-\psi}}{\int_{\Omega} e^{-\psi} d x} \quad \text { in } \Omega .
$$

We set $Q(r)=\int_{\Omega_{a}^{r}} \Delta \psi(x) d x$. Since $\psi$ is radial,

$$
\begin{aligned}
Q(r) & =\int_{\Omega_{a}^{r}} \Delta \psi(x) d x=\int_{\Omega_{a}^{r}}\left(\psi^{\prime \prime}(|x|)-\frac{d-1}{|x|} \psi^{\prime}(|x|)\right) d x \\
& =\int_{a}^{r} \int_{S(s)}\left(\psi^{\prime \prime}(s)+\frac{d-1}{s} \psi^{\prime}(s)\right) d S d s \\
& =\sigma_{d} r^{d-1} \psi^{\prime}(r)-\sigma_{d} a^{d-1} \psi^{\prime}(a) .
\end{aligned}
$$

But $\psi^{\prime}(a)=0$, and furthermore, from the right hand side of (13),

$$
Q(r)=\frac{m}{\int_{\Omega_{a}^{r}} e^{-\psi(x)} d x} \int_{a}^{r} \int_{S(s)} e^{-\psi(s)} d S d s=\lambda \int_{a}^{r} \sigma_{d} s^{d-1} e^{-\psi(s)} d s,
$$

which gives

$$
Q^{\prime}(r)=\frac{d}{d r}\left(\lambda \int_{a}^{r} \sigma_{d} s^{d-1} e^{-\psi(s)} d s\right)=\lambda \sigma_{d} r^{d-1} e^{-\psi(r)} .
$$

Multiplying both sides of (14) by $r^{1-d}$ and differentiating with respect to $r$ we obtain

$$
(1-d) r^{-d} Q^{\prime}(r)+r^{d-1} Q^{\prime \prime}(r)=-\lambda \psi^{\prime}(r) \sigma_{d} e^{-\psi(r)} .
$$

Using $\sigma_{d} r^{d-1} \psi^{\prime}(r)=Q(r)$ and multiplying (15) by $r^{d}$, we deduce from (14) and (15) the equation

$$
Q^{\prime \prime}(r)+(1-d) \frac{1}{r} Q^{\prime}(r)+\frac{1}{\sigma_{d}} r^{1-d} Q^{\prime}(r) Q(r)=0 .
$$


Moreover, this equation is supplemented by the boundary conditions

$$
\begin{aligned}
& Q(a)=\int_{\Omega_{a}^{a}} \Delta \Psi(x) d x=0, \\
& Q(A)=\int_{\Omega_{a}^{A}} \Delta \Psi(x) d x=\frac{m}{\int_{\Omega_{a}^{A}} e^{-\psi(x)} d x} \int_{\Omega_{a}^{A}} e^{-\psi(x)} d x=m .
\end{aligned}
$$

Notice that this procedure reduces our nonlocal problem to a local one.

Now, we can set

$$
\begin{aligned}
s & =\log r, \\
v(s) & =\sigma_{d}^{-1} r^{3-d} Q^{\prime}(r), \\
w(s) & =\sigma_{d}^{-1} r^{2-d} Q(r) .
\end{aligned}
$$

Then (16) will take the form of the dynamical system

$$
\left\{\begin{array}{l}
v^{\prime}(s)=(2-w) v \\
w^{\prime}(s)=(2-d) w+v
\end{array}\right.
$$

with the initial conditions

$$
\begin{aligned}
w(\log a) & =\sigma_{d}^{-1} a^{2-d} Q(a)=0, \\
w(\log A) & =\sigma_{d}^{-1} A^{2-d} Q(A)=\sigma_{d}^{-1} A^{2-d} m,
\end{aligned}
$$

resulting from (17).

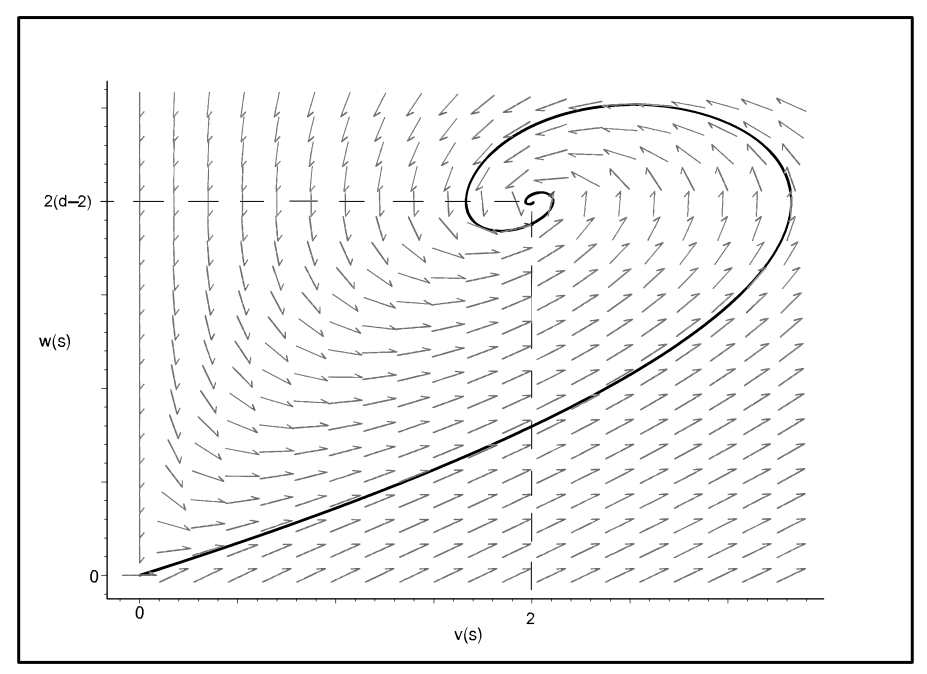

Fig. 1

Figure 1 shows the phase portrait of (18) in the first quadrant for $d=3$. In the general case $(3 \leq d \leq 9)$ the image is similar. We have two stationary points $P_{1}=\left(\begin{array}{l}0 \\ 0\end{array}\right)$ i $P_{2}=\left(\begin{array}{c}2(d-2) \\ 2\end{array}\right)$. The first one is of source type, and the second 
one is a focus. These and other properties of this system can be found for example in [5].

Let us state two simple lemmas:

LEMMA 4.2. The vector field generated by the system (18) is continuous, and transforms every interval $\{(x, 0): 0<\delta<x\}$ to some curve homeomorphic to this interval.

Lemma 4.3. For every number $q_{0}<l<q_{1}$ and every point $\left(w_{0}, 0\right)$ there exists $\Delta t$ such that the trajectory of the system (18), starting at time $t_{0}$ from $\left(w_{0}, 0\right)$, intersects the line $w=l$ at least $k$ times for every fixed $k \in \mathbb{N}$ in the time interval $\left[t_{0}, t_{0}+\Delta t\right]$.

Lemma 4.3 may be easily deduced from Figure 1 and the Lyapunov function for the system (18),

$$
L=\frac{1}{2}(w-2)^{2}+(v-2(d-2))-2(d-2) \log \left(\frac{v}{2(d-2)}\right) .
$$

Proof of Theorem 4.1. Since $P_{1}$ is a stationary point and the vector field is continuous, for any $t_{0}, \Delta t>0$ and $\varepsilon_{1}>0$ we can find $0<\varepsilon_{2} \ll 1$ such that the trajectory starting from the point $\left(\varepsilon_{2}, 0\right)$ at time $t_{0}$ will remain in the ball $B\left((0,0), \varepsilon_{1}\right)$ in the time interval $\left[t_{0}, t_{0}+\Delta t\right]$.

For simplicity, let us fix the inner radius $a$ of our annulus to be 1 . Then from (19) we have $t_{0}=0$. Now, we choose a point $\left(x_{0}, 0\right)$ on the $v$-axis and some positive number $l_{0}$ such that the line $w=l_{0}$ crosses the separatrix in at least two points. Next, we set the outer radius $A$ of our annulus such that trajectory starting from $\left(x_{0}, 0\right)$ for $t_{0}=0$ intersects the line $w=l_{0}$ twice in the time interval $[0, \Delta t]$, where $\Delta t=\log A$. Finally, we determine the parameter $m_{0}$ such that $\sigma_{d}^{-1} A^{2-d} m_{0}=l_{0}$.

As mentioned before we can now choose $(\varepsilon, 0)$ such that the trajectory starting from $(\varepsilon, 0)$ at time $t_{0}$ will not cross the line $w=l_{0}$ before time $t_{0}+\Delta t$. Now by Lemma 4.2 the interval $I=\left\{(x, 0): \varepsilon<x<x_{0}\right\}$ is transformed by the dynamical system (18) to a curve $\Gamma$, homeomorphic to this interval. This means that there exist at least two points $\mathcal{K}_{1}=\left(x_{1}^{k}, l_{0}\right)$, $\mathcal{K}_{2}=\left(x_{2}^{k}, l_{0}\right)$ such that $\Gamma$ crosses the line $w=l_{0}$ at $\mathcal{K}_{1}, \mathcal{K}_{2}$. Hence we can find two points $\mathcal{N}_{1}=\left(x_{1}^{n}, 0\right), \mathcal{N}_{2}=\left(x_{2}^{n}, 0\right)$ in $I$ such that the trajectories starting from $\mathcal{N}_{1}, \mathcal{N}_{2}$ will, after time $t_{0}+\Delta t$, stop at line $w=l_{0}$. These are the trajectories we are looking for. They represent two different solutions of (9) on the annulus $\Omega_{a}^{A}$ with parameter $m=m_{0}$.

COROllary 4.4. Taking $a, A, m$ in the proof of Theorem 4.1 more carefully, we can construct exactly $k$ different radial solutions for the problem (9) in $\Omega_{a}^{A}$ and any fixed $k \in \mathbb{N}$. 
REMARK 4.5. By applying this approach, for a fixed $m$, it is not difficult to show uniqueness of radial solutions when $A / a>0$ is sufficiently small and nonexistence of such solutions for $A / a \gg 1$.

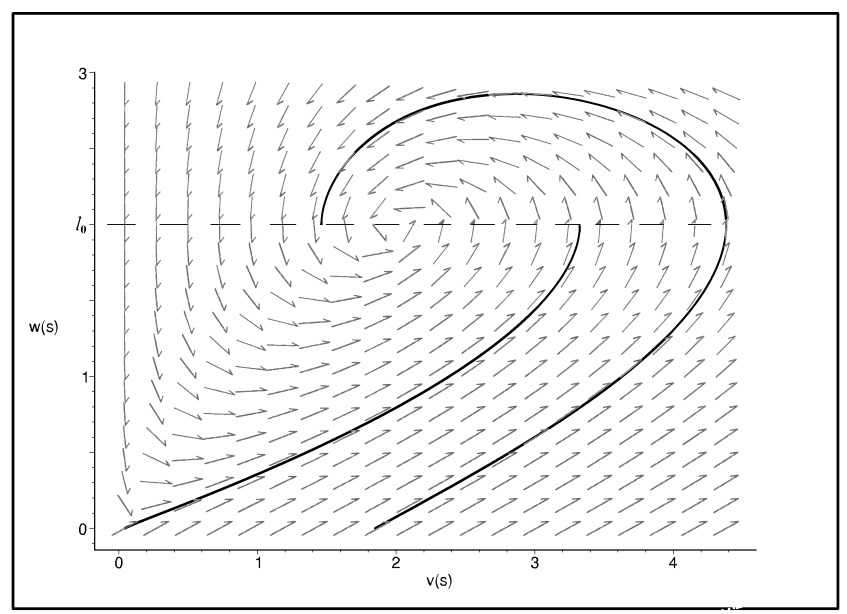

Fig. 2

Examples of such solutions can be found below. We put $r=1, R=$ $e^{3}, m=504.8424623$. After a suitable change of variables we find two trajectories such that for $t_{0}=0, t_{1}=3$ we have $w\left(t_{0}\right)=0$ and $w\left(t_{1}\right)=2.00015$ (we used the Fehlberg fourth-fifth order Runge-Kutta method). In Figure 2 we have both trajectories $(w, v)$. The left one starts from $(0.04026,0)$, and the right one from $(1.77,0)$. Figure 3 shows the section of the graph of the density function along some ray starting from $(0,0)$.

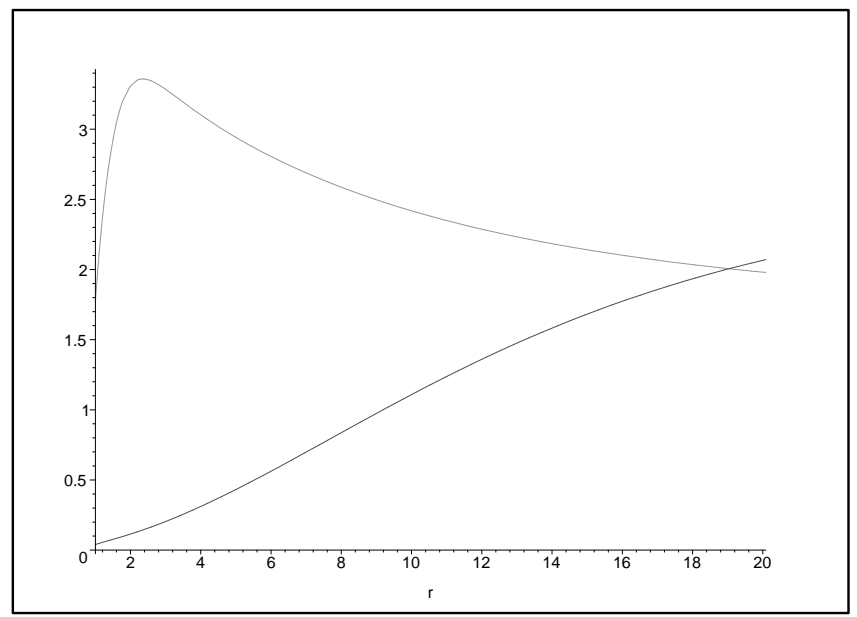

Fig. 3 


\section{References}

[1] P. Biler, J. Dolbeault, M. J. Esteban, P. Markowich and T. Nadzieja, Steady states for Streater's energy-transport models of self-gravitating particles, in: Transport in Transition Regimes, N. Ben Abdallah et al. (eds.), Springer IMA Vol. Math. Appl. 135, Springer, 2003, 37-56.

[2] P. Biler, A. Krzywicki and T. Nadzieja, Self-interaction of Brownian particles coupled with thermodynamics processes, Rep. Math. Phys. 42 (1998), 359-372.

[3] P. Biler and T. Nadzieja, Existence and nonexistence of solutions for a model of gravitational interaction of particles I, Colloq. Math. 64 (1994), 319-334.

[4] E. Caglioti, P. L. Lions, C. Marchioro and M. Pulvirenti, A special class of stationary flows for two-dimensional Euler equations: a statistical mechanics description $I$, Comm. Math. Phys. 143 (1992), 501-525.

[5] I. M. Gelfand, Some problems in the theory of quasi-linear equations, Amer. Math. Soc. Transl. 29 (1963), 295-381.

[6] T. Nagai, T. Senba and K. Yoshida, Application of the Trudinger-Moser inequality to a parabolic system of chemotaxis, Funkc. Ekvac. 40 (1997), 411-433.

[7] K. Nagasaki and T. Suzuki, Radial and nonlinear eigenvalue problem $\Delta u+\lambda e^{u}=0$ on annuli in $\mathbb{R}^{2}$, J. Differential Equations 87 (1990), 144-168.

[8] M. Smoluchowski, Drei Vorträge über Diffusion, Brownische Molekularbewegung und Koagulation von Kolloidteilchen, Phys. Z. 17 (1916), 557-571, 585-599.

[9] R. F. Streater, Dynamics of Brownian particles in a potential, J. Math. Phys. 38 (1997), 4570-4575.

Institute of Mathematics

Wrocław University

Pl. Grunwaldzki 2/4,

50-384 Wrocław, Poland

E-mail: olech@math.uni.wroc.pl 